\title{
DESEMPENHO DE DOIS SISTEMAS DISTRIBUIDORES DE SEMENTES UTILIZADOS EM SEMEADORAS DE MILHO ${ }^{1}$
}

\author{
EVANDRO CHARTUNI MANTOVANI, BARBARA HELIODORA MACHADO MANTOVANI, IVAN CRUZ², \\ WALTER LUIZ DE CASTRO MEWES ${ }^{3}$ e ANTÔNIO CARLOS DE OLIVEIRA ${ }^{2}$
}

\begin{abstract}
RESUMO - O tratamento de sementes de milho (Zea mays L.) com inseticidas altera a rugosidade da superfície das sementes, afetando o desempenho da semeadora, pela difículdade de movimentá-las no depósito e também nos sistemas distribuidores, e a densidade de plantio. Determinou-se o efeito do tratamento com inseticidas ou grafite sobre o ângulo de repouso de sementes de milho, e avaliou-se o efeito da velocidade de deslocamento, do tratamento químico e com grafite das sementes, além da classificação por peneiras, no desempenho de dois sistemas distribuidores, de disco e de dedos prensores. Os testes foram realizados em uma bancada montada em laboratório com sementes classificadas em diferentes peneiras e submetidas a seis tratamentos, testadas em duas velocidades de deslocamento e em dois sistemas de distribuição de sementes. O delineamento utilizado foi em blocos casualizados, com três repetições. No sistema de dedos prensores, o uso do inseticida melhorou significativamente o desempenho da semeadora, ao contrário do verificado no sistema de disco. Com a adição de grafite, na dose de $4 \mathrm{~g} / \mathrm{kg}$ de sementes, o desempenho voltou a ser o mesmo de antes do tratamento com a utilização de Semevin, e ligeiramente inferior com Furadan.
\end{abstract}

Termos para indexação: Zea mays, inseticida, ângulo de repouso, grafite.

\section{PERFORMANCE OF TWO SEED DISTRIBUTION MECHANISMS USED ON CORN SEEDERS}

\begin{abstract}
Corn (Zea mays L.) seed treatment with insecticide to control soil pests alters the surface roughness, difficulting seed to slide from the seed reservoir to the soil, affecting the performance of the seeder. The objectives of this work were to determine the effect of seed treatment with insecticides or graphite on the angle of repose of maize seed and to evaluate the effect of seeder velocity, chemical treatment, seed size and shape on the performance of two seed distribution systems: disc and fingers. The tests were performed in a laboratory, by using maize seeds of the cultivar BR 201, with different classifications, submitted to six chemical treatments, tested at two velocities, with two types of seed distribution system. The experiment was designed in randomized complete blocks, with three replications. All isolated factors and interactions were significant at 5\% probability level. The use of the insecticides Furadan and Semevin made the performance of the seeder become better for the finger system and worse for the disc system. Adding graphite, at the dose of $4 \mathrm{~g} / \mathrm{kg}$ of seed, improved performance to the levels before the chemical treatment, when using Semevin, and to levels slightly slower with Furadan.
\end{abstract}

Index terms: Zea mays, maize, insecticide, repose angle, graphite

\section{INTRODUÇÃO}

O escoamento das sementes de milho no depósito das semeadoras-adubadoras se faz por gravida-

\footnotetext{
${ }^{1}$ Aceito para publicação em 15 de junho de 1998.

${ }^{2}$ Eng. Agr., Ph.D., Embrapa-Centro Nacional de Pesquisa de Milho e Sorgo (CNPMS), Caixa Postal 151, CEP 35701-970 Sete Lagoas, MG. E-mail evandro@cnpms.embrapa.br

${ }^{3}$ Eng. Agr., M.Sc., UFV/CEDAF, CEP 35701-970 Florestal, MG.
}

de. A facilidade com que tal escoamento se verifica depende, entre outros fatores, do coeficiente de atrito interno do material granular e deste com as paredes do reservatório. O coeficiente de atrito entre o material granular é igual à tangente do ângulo de atrito interno para aquele material. $\mathrm{O}$ ângulo de repouso é aquele máximo do talude formado pelo material granular amontoado, em relação à horizontal. Sabe-se que esses valores, embora não sejam iguais, podem ser assumidos como tendo a mesma magnitude, para efeito de projetos de escoamento de mate- 
rial granular. Os fatores que afetam o ângulo de repouso são o tamanho e a forma das sementes, o teor de umidade, a presença de material estranho, a orientação das partículas e, principalmente, a rugosidade da superfície da semente (Mohsenin, 1974).

Tem-se observado que o tratamento químico de sementes de milho, geralmente com inseticidas, utilizado para combater pragas de solo durante o plantio, altera a rugosidade da superfície delas, pelo aumento do ângulo de repouso, afetando o desempenho da semeadora, pela dificuldade de movimentação no depósito e também nos sistemas distribuidores (discos ou dedos prensores). Tal fato tem um efeito direto no estabelecimento do estande ideal para a cultura do milho.

A partir do final da década de 80, as empresas produtoras de sementes começaram a comercializar híbridos com características de materiais modernos, para melhorar a produtividade, com espigas que apresentam sabugo fino e grãos longos, em forma de cunha. Um exemplo desses materiais é o híbrido da Embrapa BR 201, cujas sementes das peneiras 18 e 20 apresentam maior razão entre o comprimento e a largura, e menor espessura que a normalmente encontrada em sementes do mesmo tamanho, de outras cultivares, o que dificulta o seu escoamento no depósito.

Uma maneira de contornar esse problema de escoamento pode ser o uso de uma substância inerte lubrificante, como o grafite, que diminua tanto o coeficiente de atrito entre as sementes como destas com as paredes do reservatório. Isso é feito empiricamente, sem levar em conta a quantidade a ser usada e o efeito do uso do grafite nos diferentes sistemas de distribuição de sementes.

Há controvérsias quanto ao efeito do nível de sementes no depósito sobre a precisão de distribuição delas no plantio. Chhinnan et al. (1975) obtiveram um aumento no número de capturas múltiplas de sementes pelas células com a diminuição do nível de sementes no reservatório. Por sua vez, Moreira et al. (1978) e Gazzola (1989) afirmaram que o nível das sementes não influenciou significativamente na precisão de dosagem e na uniformidade de distribuição longitudinal dos grãos.

O aumento da velocidade de deslocamento modifica a velocidade periférica do disco, ocasionando danos mecânicos às sementes e comprometendo a ocupação das células e dos dedos prensores. Delafosse (1986) afirma que, em quase todos os sistemas de semeaduras conhecidos, a qualidade da semeadura diminui quando se aumenta a velocidade de trabalho. Segundo Barmington (1948), velocidades altas nos distribuidores causam grande número de células vazias, e em baixas velocidades elevado número de capturas múltiplas de sementes pelo distribuidor. Delafosse (1986) recomenda, para determinadas espécies de sementes e tipos de distribuidores, o uso de velocidades periféricas máximas entre 0,29 e $0,315 \mathrm{~m} / \mathrm{s}$, para uma boa precisão de dosagem. Roth \& Porterfield (1960) relatam que à medida que se aumentam as tolerâncias dadas às dimensões das células distribuidoras, aumenta-se a velocidade periférica do distribuidor, mantendo-se $100 \%$ de enchimento das células.

Kepner et al. (1978) afirmam que o diâmetro das células distribuidoras deve ser $10 \%$ maior que a máxima dimensão de sementes empregadas, e a espessura, igual ao diâmetro médio ou espessura média da semente.

O objetivo deste trabalho foi determinar o efeito de grafite e de inseticidas no ângulo de repouso de sementes de milho, e avaliar o efeito da velocidade de deslocamento, do tamanho da semente e do tratamento químico e com grafite no desempenho de dois sistemas distribuidores, de disco e de dedos prensores.

\section{MATERIAL E MÉTODOS}

O trabalho foi realizado no laboratório de mecanização da Embrapa-Centro Nacional de Pesquisa de Milho e Sorgo, em Sete Lagoas, MG. Foram realizados testes, de bancadas, em dois pontos da unidade de distribuição de sementes: no depósito, para estudar o escoamento, e no distribuidor. Em ambos foram utilizadas sementes de milho da cultivar BR 201, classificadas por tamanho, nas peneiras $18 \mathrm{C}, 18 \mathrm{~L}, 20 \mathrm{C}, 20 \mathrm{~L}, 22 \mathrm{C}, 22 \mathrm{~L}$ e 24, e tratadas com grafite (G), Semevin (S), Semevin+grafite (SG), Furadan (F) e Furadan+grafite (FG), incluindo também uma amostra testemunha $(\mathrm{T})$.

O ângulo de repouso foi determinado em um experimento com delineamento experimental inteiramente casualizado, com três repetições. Uma amostra de $5 \mathrm{~kg}$ de sementes foi colocada em uma caixa com as dimensões de 0,15 X 0,30 X 0,60 m. Um dos lados da caixa constituía-se 
de uma comporta que, ao ser aberta, permitia o escoamento dos grãos. O ângulo de repouso é dado pelo $\operatorname{arc} \operatorname{tg} a / b$, sendo $a$ a abertura dada na comporta e $b$ a distância até onde a semente escoa na horizontal.

A quantidade de grafite utilizada no tratamento foi determinada misturando-se grafite à amostra, em quantidades crescentes de $1 \mathrm{~g} / \mathrm{kg}$ de semente, repetindo-se a operação até que os valores do ângulo de repouso não mais variassem.

Os testes no distribuidor de sementes foram realizados em uma bancada composta de: motor elétrico com regulador de velocidade, dois sistemas de distribuição de sementes, de disco e de dedos prensores, um eixo cardan para conexão do mecanismo dos distribuidores ao motor elétrico e um sistema de coleta automática de dados, com sensores acoplados, para monitoramento das voltas dos discos. As sementes foram avaliadas em dois sistemas de distribuição, com duas velocidades de deslocamento, $5 \mathrm{~km} / \mathrm{h}$ e $7 \mathrm{~km} / \mathrm{h}$, cada um deles constituindo-se num experimento, com delineamento em blocos casualizados e três repetições.

O desempenho foi medido pela porcentagem de células do disco ou de dedos prensores que eram preenchidos por sementes em cada volta. No caso de caírem mais sementes do que o número de furos do disco ou o número de dedos prensores, o desempenho era considerado menor que $100 \%$, tendo em vista que a queda de duplos também é um fato indesejado. O desempenho é, nesse caso, igual a 100 - (\% queda - 100)/2.

\section{RESULTADOS E DISCUSSÃO}

Os testes preliminares indicaram uma dose de, no mínimo, $4 \mathrm{~g}$ de grafite/ $\mathrm{kg}$ de sementes para atingir o menor ângulo de repouso possível. Esse resultado é importante para que se volte sempre ao mesmo ângulo de repouso da semente no depósito da semeadora, quando tratada com inseticida, em relação à semente não tratada, evitando-se a formação de vazios que impedem a alimentação dos discos de plantio.

A Tabela 1 mostra as médias gerais dos ângulos de repouso em relação aos tratamentos. O coeficiente de variação do experimento foi $1,76 \%$. Pela análise de variância, observou-se que houve significância dos fatores tamanho de sementes e tratamento de sementes com grafite, e também da interação entre eles, no nível de $5 \%$ de probabilidade. Comparando-se as médias das colunas T e G, que correspondem respectivamente à testemunha $(\mathrm{T})$ e ao tratamento semente com grafite $(\mathrm{G})$, observa-se que de fato o grafite exerce uma função significativa de lubrificante, diminuindo o ângulo de repouso das sementes de todas as peneiras. Da mesma forma, esse efeito é comprovado quando se observam, duas a duas, as colunas S e SG (Semevin e Semevin + grafite) e F e FG (Furadan e Furadan + grafite). Pode-se observar, ainda, que o tratamento com Furadan (F) teve o mesmo efeito sobre o ângulo de repouso que o tratamento com Semevin (S) em todas as peneiras, com exceção da 20C, com Furadan. O uso do grafite nas sementes tratadas com inseticida (FG e SG) fez o ângulo de repouso voltar a valores iguais ou menores do que os da testemunha (T) sem inseticida ou grafite.

Observa-se que há diferenças entre os ângulos de repouso em virtude do tamanho de sementes; entretanto, o efeito se apresenta diferenciado com relação aos tratamentos utilizados.

A análise de variância dos dados de enchimento de células revelou que os fatores isolados e as interações foram significativas a $5 \%$ de probabilidade, exceto a interação entre velocidade e peneira, no sistema de disco. O coeficiente de variação neste sistema foi $2,63 \%$, enquanto no de dedos prensores foi de $2,11 \%$.

Na Tabela 2 são apresentadas as médias do desempenho do sistema distribuidor de discos, em função da velocidade de deslocamento, da classificação e do tratamento das sementes. A porcentagem de enchimento de células, em média, foi melhor na menor velocidade $(5 \mathrm{~km})$, excetuando o tratamento FG. Entretanto, tais valores estão dentro da faixa aceitável para enchimento de células. Independentemente do tratamento químico e da velocidade de trabalho, as sementes tratadas com grafite apresentaram melhor desempenho.

Comparando-se os dados das colunas T e G, observa-se que não houve diferença estatística entre as médias dos tratamentos da testemunha e da testemunha com grafite, com sementes menores. No caso das sementes de peneiras 20L, 22L e 24, o desempenho piorou com a adição de grafite. O tratamento com Furadan ou Semevin piora o desempenho (comparando-se T com S e T com F) e, de modo geral, este resultado é acentuado com a adição de Furadan. Com a adição de grafite na semente tratada com inseticida, o desempenho volta a ser o mesmo de antes do 
tratamento (T), quando conjugado com Semevin, e ligeiramente inferior com Furadan, no caso de peneiras maiores (22L e 24$)$, na velocidade de $5 \mathrm{~km} / \mathrm{h}$, e peneiras menores, a $7 \mathrm{~km} / \mathrm{h}$.
Na Tabela 3 são apresentadas as médias do desempenho do sistema distribuidor de dedos prensores, em função da velocidade de deslocamento e do tratamento das sementes. Ao contrário do

TABELA 1. Ângulo de repouso de sementes de milho tratadas com inseticidas e com grafite, em relação à classificação por peneiras. Embrapa-Centro Nacional de Pesquisa de Milho e Sorgo (CNPMS), Sete Lagoas, MG, 1993'.

\begin{tabular}{|c|c|c|c|c|c|c|}
\hline \multirow[t]{2}{*}{ Peneira } & \multicolumn{6}{|c|}{ Tratamento $^{2}$} \\
\hline & $\mathrm{T}$ & G & $\mathrm{S}$ & SG & $\mathrm{F}$ & FG \\
\hline $18 \mathrm{C}$ & $35,5 \mathrm{bB}$ & $29,6 \mathrm{aD}$ & $39,8 \mathrm{eA}$ & $34,2 \mathrm{bcC}$ & $40,6 \mathrm{dA}$ & $35,3 \mathrm{aB}$ \\
\hline $18 \mathrm{~L}$ & $34,4 \mathrm{cB}$ & $26,6 \mathrm{bD}$ & $42,3 \mathrm{bcA}$ & $34,7 \mathrm{bB}$ & $42,9 \mathrm{bcA}$ & $29,0 \mathrm{cC}$ \\
\hline $20 \mathrm{C}$ & $37,8 \mathrm{aC}$ & $26,6 \mathrm{bE}$ & $41,0 \mathrm{dA}$ & $29,5 \mathrm{fD}$ & $39,3 \mathrm{eB}$ & $28,7 \mathrm{cdD}$ \\
\hline $20 \mathrm{~L}$ & $31,0 \mathrm{dC}$ & $26,6 \mathrm{bE}$ & 42,9abA & $33,5 \mathrm{cdB}$ & $43,8 \mathrm{abA}$ & $28,2 \mathrm{cdD}$ \\
\hline $22 \mathrm{C}$ & $34,6 \mathrm{bcC}$ & $29,7 \mathrm{aE}$ & $43,4 \mathrm{aA}$ & $36,9 \mathrm{aB}$ & $41,9 \mathrm{cA}$ & $34,2 \mathrm{bC}$ \\
\hline $22 \mathrm{~L}$ & $34,2 \mathrm{cB}$ & $27,4 \mathrm{bE}$ & $42,9 \mathrm{abA}$ & $31,3 \mathrm{eC}$ & $42,9 \mathrm{bcA}$ & $29,0 \mathrm{cD}$ \\
\hline 24 & $37,8 \mathrm{aB}$ & $26,9 \mathrm{bE}$ & $43,4 \mathrm{aA}$ & $32,7 \mathrm{dC}$ & $44,0 \mathrm{aA}$ & $27,9 \mathrm{dD}$ \\
\hline Média & $35,15 \mathrm{~B}$ & $27,6 \mathrm{E}$ & $42,0 \mathrm{~A}$ & $33,3 \mathrm{C}$ & $42,2 \mathrm{~A}$ & $30,3 \mathrm{D}$ \\
\hline
\end{tabular}

TABELA2. Porcentagem de células preenchidas por sementes no sistema de distribuição de disco, em função da velocidade da semeadora, do tamanho da semente (peneira) e do tratamento. Embrapa-Centro Nacional de Pesquisa de Milho e Sorgo (CNPMS), Sete Lagoas, MG, $1991^{1}$.

\begin{tabular}{|c|c|c|c|c|c|c|c|}
\hline \multirow[t]{2}{*}{ Peneira } & \multicolumn{6}{|c|}{ Tratamento $^{2}$} & \multirow[t]{2}{*}{ Média } \\
\hline & $\mathrm{T}$ & G & $S$ & SG & $\mathrm{F}$ & FG & \\
\hline \multicolumn{8}{|c|}{ Velocidade de $5 \mathrm{~km} / \mathrm{h}$} \\
\hline $18 \mathrm{C}$ & $97,3 \mathrm{~A}$ & $97,2 \mathrm{~A}$ & $86,7 \mathrm{~B}$ & $98,5 \mathrm{~A}$ & $80,7 \mathrm{C}$ & $97,3 \mathrm{~A}$ & $92,9 d$ \\
\hline $18 \mathrm{~L}$ & $98,0 \mathrm{AB}$ & $98,8 \mathrm{~A}$ & $91,3 \mathrm{C}$ & $97,3 \mathrm{AB}$ & $90,7 \mathrm{C}$ & $96,7 \mathrm{~B}$ & $95,5 b$ \\
\hline $20 \mathrm{C}$ & $99,2 \mathrm{AB}$ & $99,3 \mathrm{~A}$ & $98,8 \mathrm{AB}$ & $97,2 \mathrm{~B}$ & $89,3 \mathrm{C}$ & $97,2 \mathrm{~B}$ & $96,8 \mathrm{a}$ \\
\hline $20 \mathrm{~L}$ & $96,7 \mathrm{~B}$ & $99,3 \mathrm{~A}$ & $86,3 \mathrm{C}$ & $96,7 \mathrm{~B}$ & $80,7 \mathrm{D}$ & $95,3 \mathrm{~B}$ & $92,5 \mathrm{~d}$ \\
\hline $22 \mathrm{C}$ & $97,3 \mathrm{~A}$ & $99,2 \mathrm{~A}$ & $88,7 \mathrm{~B}$ & $97,7 \mathrm{~A}$ & $83,3 \mathrm{C}$ & $98,3 \mathrm{~A}$ & $94,1 \mathrm{c}$ \\
\hline $22 \mathrm{~L}$ & $96,8 \mathrm{~A}$ & $94,2 \mathrm{~B}$ & $70,0 \mathrm{E}$ & $96,0 \mathrm{AB}$ & $82,7 \mathrm{C}$ & $79,2 \mathrm{D}$ & $86,5 f$ \\
\hline 24 & $99,2 \mathrm{~A}$ & $92,7 \mathrm{~B}$ & $86,7 \mathrm{C}$ & $98,8 \mathrm{~A}$ & $78,7 \mathrm{D}$ & $94,3 \mathrm{~B}$ & $91,7 \mathrm{e}$ \\
\hline Média & $97,8 \mathrm{~A}$ & $97,2 \mathrm{~B}$ & $86,9 \mathrm{D}$ & $97,5 \mathrm{AB}$ & $83,7 \mathrm{E}$ & $94,1 \mathrm{C}$ & \\
\hline \multicolumn{8}{|c|}{ Velocidade de $7 \mathrm{~km} / \mathrm{h}$} \\
\hline $18 \mathrm{C}$ & $98,8 \mathrm{~A}$ & $98,3 \mathrm{~A}$ & $86,3 \mathrm{C}$ & $96,0 \mathrm{~B}$ & $73,7 \mathrm{D}$ & $96,3 \mathrm{~B}$ & $91,6 \mathrm{~d}$ \\
\hline $18 \mathrm{~L}$ & $97,3 \mathrm{AB}$ & $99,2 \mathrm{~A}$ & $89,3 \mathrm{C}$ & $96,3 \mathrm{~B}$ & $87,0 \mathrm{D}$ & $97,7 \mathrm{C}$ & $94,5 b$ \\
\hline $20 \mathrm{C}$ & $98,0 \mathrm{~A}$ & $97,8 \mathrm{~A}$ & $91,3 \mathrm{~B}$ & $97,7 \mathrm{~A}$ & $90,3 \mathrm{~B}$ & $98,5 \mathrm{~A}$ & $95,6 a$ \\
\hline $20 \mathrm{~L}$ & $95,0 \mathrm{~B}$ & $98,0 \mathrm{~A}$ & $85,7 \mathrm{C}$ & $93,3 \mathrm{~B}$ & $74,7 \mathrm{D}$ & $95,0 \mathrm{~B}$ & $90,3 \mathrm{e}$ \\
\hline $22 \mathrm{C}$ & $96,0 \mathrm{~B}$ & $97,3 \mathrm{AB}$ & $84,7 \mathrm{C}$ & $98,3 \mathrm{~A}$ & $83,3 \mathrm{C}$ & $98,0 \mathrm{~B}$ & $92,9 \mathrm{c}$ \\
\hline $22 \mathrm{~L}$ & $89,7 \mathrm{~B}$ & $85,2 \mathrm{C}$ & $58,7 \mathrm{E}$ & $93,0 \mathrm{~A}$ & $77,3 \mathrm{D}$ & $92,0 \mathrm{~A}$ & $82,6 f$ \\
\hline 24 & $97,9 \mathrm{~A}$ & $95,7 \mathrm{~B}$ & $82,0 \mathrm{C}$ & $99,0 \mathrm{~A}$ & $78,3 \mathrm{D}$ & $95,2 \mathrm{~B}$ & $91,3 \mathrm{~d}$ \\
\hline Média & $96,1 \mathrm{~A}$ & $95,9 \mathrm{~A}$ & $82,6 \mathrm{~B}$ & $96,2 \mathrm{~A}$ & $80,7 \mathrm{C}$ & $96,1 \mathrm{~A}$ & \\
\hline
\end{tabular}


TABELA3. Porcentagem de células preenchidas no sistema distribuidor de dedos prensores (\%), em função da velocidade da semeadora, do tamanho das sementes (peneira) e do tratamento de sementes. Embrapa-Centro Nacional de Milho e Sorgo (CNPMS), Sete Lagoas, MG, 1991.

\begin{tabular}{|c|c|c|c|c|c|c|c|}
\hline \multirow[t]{2}{*}{ Peneira } & \multicolumn{6}{|c|}{ Tratamento $^{2}$} & \multirow[t]{2}{*}{ Média } \\
\hline & $\mathrm{T}$ & G & $\mathrm{S}$ & $\mathrm{SG}$ & $\mathrm{F}$ & $\mathrm{FG}$ & \\
\hline \multicolumn{8}{|c|}{ Velocidade de $5 \mathrm{~km} / \mathrm{h}$} \\
\hline $18 \mathrm{C}$ & $95,3 \mathrm{~B}$ & $92,7 \mathrm{C}$ & $96,6 \mathrm{C}$ & $87,0 \mathrm{D}$ & $99,2 \mathrm{~A}$ & $92,0 \mathrm{C}$ & $93,1 \mathrm{a}$ \\
\hline $18 \mathrm{~L}$ & $90,7 \mathrm{C}$ & $88,3 \mathrm{D}$ & $98,3 \mathrm{~A}$ & $93,0 \mathrm{~B}$ & $98,0 \mathrm{~A}$ & $88,7 \mathrm{D}$ & $92,8 \mathrm{a}$ \\
\hline $20 \mathrm{C}$ & $89,0 \mathrm{~B}$ & $87,3 \mathrm{BC}$ & $99,3 \mathrm{~A}$ & $86,0 \mathrm{CD}$ & $97,9 \mathrm{~A}$ & $84,3 \mathrm{D}$ & $90,6 b$ \\
\hline $20 \mathrm{~L}$ & $86,0 \mathrm{C}$ & $86,3 \mathrm{C}$ & $97,7 \mathrm{~A}$ & $89,0 \mathrm{~B}$ & $98,2 \mathrm{~A}$ & $83,3 \mathrm{D}$ & $90,1 \mathrm{c}$ \\
\hline $22 \mathrm{C}$ & $91,3 \mathrm{~B}$ & $89,7 \mathrm{BC}$ & $95,5 \mathrm{~A}$ & $96,0 \mathrm{~A}$ & $97,2 \mathrm{~A}$ & $88,0 \mathrm{C}$ & $92,9 \mathrm{a}$ \\
\hline $22 \mathrm{~L}$ & $92,7 \mathrm{~B}$ & $84,7 \mathrm{C}$ & $98,8 \mathrm{~A}$ & $76,3 \mathrm{E}$ & $97,5 \mathrm{~A}$ & $81,0 \mathrm{D}$ & $88,5 \mathrm{~d}$ \\
\hline 24 & $88,0 \mathrm{~B}$ & $78,0 \mathrm{D}$ & $98,3 \mathrm{~A}$ & $81,0 \mathrm{C}$ & $99,0 \mathrm{~A}$ & $73,3 \mathrm{E}$ & $86,3 \mathrm{e}$ \\
\hline Média & $90,4 \mathrm{C}$ & $86,7 \mathrm{D}$ & $97,2 \mathrm{~B}$ & $86,9 \mathrm{D}$ & $98,1 \mathrm{~A}$ & $84,4 \mathrm{E}$ & \\
\hline \multicolumn{8}{|c|}{ Velocidade de $7 \mathrm{~km} / \mathrm{h}$} \\
\hline $18 \mathrm{C}$ & $91,55 \mathrm{BC}$ & $96,95 \mathrm{CD}$ & $98,8 \mathrm{~A}$ & $94, \mathrm{~S} 3 \mathrm{D}$ & $97,5 \mathrm{AB}$ & $97,3 \mathrm{AB}$ & $96,6 \mathrm{a}$ \\
\hline $18 \mathrm{~L}$ & 93,0 & $91,0 \mathrm{C}$ & $97,3 \mathrm{~A}$ & $96,3 \mathrm{~A}$ & $97,5 \mathrm{~A}$ & $92,7 \mathrm{BC}$ & $94,6 \mathrm{c}$ \\
\hline $20 \mathrm{C}$ & $85,7 \mathrm{C}$ & $87,3 \mathrm{C}$ & $97,8 \mathrm{~A}$ & $89,3 \mathrm{~B}$ & $96,5 \mathrm{~A}$ & $90,3 \mathrm{~B}$ & $91,2 \mathrm{~d}$ \\
\hline $20 \mathrm{~L}$ & $88,0 \mathrm{E}$ & $90,0 \mathrm{CD}$ & $96,5 \mathrm{~A}$ & $91,3 \mathrm{C}$ & $93,8 \mathrm{~B}$ & $89,3 \mathrm{DE}$ & $91,5 \mathrm{~d}$ \\
\hline $22 \mathrm{C}$ & $95,0 \mathrm{~B}$ & $91,0 \mathrm{C}$ & $98,2 \mathrm{~A}$ & $98,3 \mathrm{~A}$ & $97,2 \mathrm{~A}$ & $94,7 \mathrm{~B}$ & $95,7 b$ \\
\hline $22 \mathrm{~L}$ & $94,7 \mathrm{~A}$ & $85,3 \mathrm{C}$ & $95,3 \mathrm{~A}$ & $82,7 \mathrm{D}$ & $92,5 \mathrm{~B}$ & $87,0 \mathrm{C}$ & $89,6 \mathrm{e}$ \\
\hline 24 & $96,0 \mathrm{~A}$ & $82,0 \mathrm{D}$ & $92,2 \mathrm{~B}$ & $85,3 \mathrm{C}$ & $95,33 \mathrm{~A}$ & $81,0 \mathrm{D}$ & $88,6 \mathrm{f}$ \\
\hline Média & $92,7 \mathrm{C}$ & $88,9 \mathrm{~F}$ & $96,6 \mathrm{~A}$ & $91,1 \mathrm{D}$ & $95,8 \mathrm{~B}$ & $90,3 \mathrm{E}$ & \\
\hline
\end{tabular}

que foi discutido com relação ao sistema de disco, o aumento na velocidade de deslocamento melhorou o desempenho, excetuando os casos em que as sementes foram tratadas com S ou com $\mathrm{F}$. Comparando-se os tratamentos T, F e S, observa-se que o uso do inseticida melhora significativamente o desempenho da semeadora, exceto no 18C (Semevin). Já o tratamento com grafite piora o desempenho em todas as situações, como pode ser verificado comparando-se duas a duas as colunas T e G, F e FG e S e $\mathrm{SG}, \mathrm{em}$ ambas as velocidades.

\section{CONCLUSÕES}

1. A dose de grafite indicada para uso no depósito de sementes é de, no mínimo, $4 \mathrm{~g} / \mathrm{kg}$ de sementes.

2. A melhor velocidade de plantio para o sistema de disco é de $5 \mathrm{~km} / \mathrm{h}$, e para o sistema de dedos prensores, $7 \mathrm{~km} / \mathrm{h}$.
3. O uso de inseticida nas sementes melhora significativamente o desempenho da semeadora com sistema de dedos prensores.

4. O uso de inseticida nas sementes, em sistema de distribuição de disco, dificulta o enchimento da célula e, conseqüentemente, o desempenho desse sistema.

\section{REFERÊNCIAS}

BARMINGTON, R.D. The relation of seed, cell size and speed to beet planter performance. Agricultural Engineering, St Joseph, v.28, n.2, p.49-54, 1948.

CHHINNAN, M.S.; YOUNG, J.H.; ROHRBACK, R.P. Accuracy of seed spacing in peanuts planting. Transactions of the ASAE, St Joseph, v.18, n.5, p. 828-831, 1975. 
DELAFOSSE, R.M. Máquinas sembradoras de grano grueso; descripción y uso. Santiago de Chile: FAO, 1986. $48 p$.

GAZZOLA, O. Projeto e avaliação de um sistema dosador de precisão para semeadora a tração animal. Campinas: UNICAMP, Fac. de Engenharia Agrícola, 1989. 103p. Tese de Mestrado.

KEPNER, R.A.; BAYNER, R.; BARGER, E.L. Principles of farm machinery. New York: John Wiley, 1978. $57 \mathrm{p}$.
MOHSENIN, N.N. Physical properties of plant and animal materials. New York: Gordan and Breach, 1974. $734 \mathrm{p}$.

MOREIRA, C.A.; PEREIRA, J.C.V.N.A.; MENEZES, J.F. de; COSTA, J.A. de S. Desempenho de mecanismos dosadores-distribuidores de sementes em plantadeiras-adubadeiras. Campinas: IAC, 1978. 22p. (IAC. Circular, 90).

ROTH, L.O.; PORTERFIELD, J.G. Some basic performance characteristics of a horizontal plant seed metering device. Transactions of the ASAE, v.3, n.2, p.105-107, 1960. 